AIAA-2002-1517

\title{
Finite Element Modeling of the Buckling Response of Sandwich Panels
}

\author{
Cheryl A. Rose ${ }^{1}$ \\ NASA Langley Research Center \\ Hampton, VA 23681 \\ David F. Moore \\ Lockheed-Martin Engineering and Sciences Company \\ Hampton, VA 23681 \\ Norman F. Knight, Jr. ${ }^{3}$ \\ Veridian Systems Division \\ Chantilly, VA 20151 \\ Charles C. Rankin ${ }^{4}$ \\ Lockheed-Martin Advanced Technology Center \\ Palo Alto, CA 94304
}

\begin{abstract}
A comparative study of different modeling approaches for predicting sandwich panel buckling response is described. The study considers sandwich panels with anisotropic face sheets and a very thick core. Results from conventional analytical solutions for sandwich panel overall buckling and face-sheet-wrinkling type modes are compared with solutions obtained using different finite element modeling approaches. Finite element solutions are obtained using layered shell element models, with and without transverse shear flexibility, layered shell/solid element models, with shell elements for the face sheets and solid elements for the core, and sandwich models using a recently developed specialty sandwich element. Convergence characteristics of the shell/solid and sandwich element modeling approaches with respect to in-plane and through-thethickness discretization, are demonstrated. Results of the study indicate that the specialty sandwich element provides an accurate and effective modeling approach
\end{abstract}

for predicting both overall and localized sandwich panel buckling response. Furthermore, results indicate that anisotropy of the face sheets, along with the ratio of principle elastic moduli, affect the buckling response and these effects may not be represented accurately by analytical solutions. Modeling recommendations are also provided.

\section{Introduction}

The increased performance requirements of future aeronautical and aerospace vehicles, and the projected increased demand for air travel, suggest that more efficient aeronautical and aerospace flight vehicle concepts are needed. An example of a revolutionary concept for an efficient, large transport aircraft is a blended-wing-body type (BWB) of aircraft, which blends the wings and fuselage into a single lifting surface. Due to the shape of the BWB airplane, the pressurized centerbody region, which includes both the passenger area and the cargo area, is non-circular. The

\footnotetext{
1 Aerospace Engineer, Mechanics and Durability Branch. Member AIAA.

${ }^{2}$ Aerospace Engineer. Member ASME.

${ }^{3}$ Staff Scientist. Associate Fellow AIAA. Member ASME. Corresponding author.

${ }^{4}$ Staff Scientist. Associate Fellow AIAA.

Copyright (C2002 by the American Institute of Aeronautics and Astronautics, Inc. No copyright is asserted in the United States under Title 17, U. S. Code. The U. S. Government has a royalty-free license to exercise all rights under the copyright claimed herein for Governmental Purposes. All other rights are reserved by the copyright owner.
} 
non-circular centerbody region is challenging from the standpoint of structural design since the basic cover panel structure carries both internal transverse pressure load and normal wing bending and torsion loads. In order to satisfy the performance and weight requirements for the BWB aircraft, and other advanced concepts that are subjected to bending or pressure loadings, advanced sandwich-type constructions with composite material face sheets and relatively thick cores (see Figure 1) offer a potential design advantage over conventional metallic materials and stiffened skin construction [1].

Most sandwich structures are defined using a three-layer type of construction, as illustrated in Figure 1. The outer layers are thin, stiff, high-strength material; while the middle layer is a thick, weak, low-density material. Initial analytical work on sandwich structures treated the three-dimensional sandwich structure as a pair of membrane face sheets held apart by a core material with a relatively large transverse shear stiffness. The bending stiffness of the face sheets is ignored and the core is assumed to be inextensional in the transverse direction and has negligible stiffness in the in-plane directions. This type of model, called a sandwich of the first kind, has been applied successfully in many applications. However, a more robust formulation with additional fidelity is needed to model complex nonlinear structural behavior including local failures such as face-sheet buckling and face-sheet disbond. A sandwich of the second kind accounts for the out-of-plane response of the face sheets and the full three-dimensional behavior of the core material.

Early analysis work for metallic sandwich structures includes Plantema [2], and design guidelines for sandwich structures are given in Ref. [3]. General instability and face-sheet wrinkling are described by Benson and Mayers [4]. One aspect of their work was related to defining a stability boundary between facesheet wrinkling and general instability. Researchers have also studied the analysis of sandwich structures with emphasis on the use of composite material face sheets and foam cores. Finite element formulations for sandwich panels are reviewed by $\mathrm{Ha}[5,6]$. Displacement-based formulations and hybrid formulations are considered as well as different throughthe-thickness kinematic models. No numerical studies are presented. Frostig [7] investigated sandwich panel buckling using a higher-order theory which accounts for different boundary conditions on the upper and lower face sheets. Using a closed-form solution, he studied the influence on buckling of different boundary conditions for various panel aspect ratios and for both soft and stiff cores. Tessler et al. [8] present a $\{1,2\}$-order theory accounting for transverse shear and normal stresses and strains. They present linear stress solutions for thermally loaded, simply supported sandwich plates with laminated face sheets using an analytic, closedform solution. Vonanch and Rammerstorfer [9] presented a Rayleigh-Ritz solution for face-sheet wrinkling of general unsymmetric sandwich panels with orthotropic face sheets. Comparison of their analytical results with unit-cell three-dimensional finite element results is used to verify their approach.

Bert [10] summarized different theories for sandwich plates with laminated composite face sheets that account for both transverse shear and transverse normal effects. Noor et al. [11] presented an exhaustive reference list (over 1300 citations) of analytical and computational procedures for sandwich structures. Librescu and Hause [12] presented a further survey and extended the formulation to include buckling and postbuckling response of flat and curved sandwich structures subjected to mechanical and thermal loads. A RayleighRitz procedure for simply supported sandwich plates was developed by Rao [13] where the bending stiffness of the face sheets was ignored. Kim and Hong [14] extended Rao's work to account for the face-sheet bending stiffness. Hadi and Matthews [15] presented a Rayleigh-Ritz procedure based on a zigzag theory and accounts for shear deformation in the face sheets. Comparisons with other Rayleigh-Ritz solutions were presented for sandwich panels with thin face sheets. Results are reported for different face-sheet stacking sequences. Dawe and Yuan $[16,17]$ presented a finite strip formulation using B-splines for sandwich panels with anisotropic face sheets. However, no comparative numerical studies of different finite element modeling strategies for predicting the buckling response of sandwich panels have been identified in the literature.

The present paper describes the basic buckling behavior and response of sandwich panels loaded in axial compression and compares buckling predictions for various levels of finite element modeling fidelity. Buckling results obtained from approximate analytical expressions are also compared with buckling results obtained from the finite element analyses. Different finite element models of the sandwich panel are considered including layered shell models, specialty sandwich element models, and layered shell/solid models. Numerical results obtained using the STAGS (STructural Analysis of General Shells) nonlinear finite element code [18] for the three different finite element modeling approaches are presented for selected sandwich panel design parameters. Parameters varied in the study include the face-sheet thickness and the core thickness as well as modeling fidelity. Particular attention is given to examining the buckling behavior for a specific panel aspect ratio, as the core thickness 
becomes large. The results presented demonstrate the interplay between finite element models with different levels of fidelity and the modeling requirements for accurate prediction of sandwich panel buckling response. General modeling guidelines are provided.

\section{Sandwich Panel Buckling}

Sandwich panel buckling involves several possible modes. Three buckling modes are considered in this study. One mode is an overall panel buckling mode or general instability mode where the face sheets and the core buckle together into long wavelength buckles - long in the sense that the length of a half-wave is equal to one of the planar dimensions of the sandwich panel. Another possible buckling mode consists of short wavelength buckles where the face sheets and the core do not exhibit any transverse extension through the thickness of the sandwich. This mode is a short wavelength panel-buckling mode, and is commonly referred to as an asymmetrical wrinkling mode. Another short wavelength buckling mode is one where the core material is either stretched or compressed. This mode is a short wavelength face-sheet-wrinkling mode, and is referred to as a symmetrical wrinkling mode. Other sandwich panel failure modes, such as face-sheet dimpling, core crushing, and face-sheet disbond, are not included in the present study.

Analytical predictions of overall sandwich panel buckling often ignore the bending stiffness of the core and assume that the core serves primarily to move the face sheets away from the midsurface of the panel. A simple approach for predicting overall panel buckling of a sandwich panel with homogeneous, isotropic face sheet and core materials loaded by in-plane compression and with simply supported conditions on all edges is presented by Brush and Almroth [19]. Vinson [20] developed an analytical model that includes the effects of shear deformation for predicting overall panel buckling of composite sandwich panels with orthotropic face sheets. This model, incorporated into PANDA2 [21], is used herein as the analytical model for general instability and local face-sheet wrinkling predictions.

Buckling loads for general and face-sheet-wrinkling instabilities are computed for a given sandwich configuration to determine the minimum buckling load and the critical buckling mode shape for the sandwich panel. Parameters having a significant influence on the buckling response are the face-sheet thickness, $t_{f}$, the core thickness, $h_{c}$, and the core shear stiffness, $G_{c}$ as well as the panel planar dimensions $a$ and $b$. Researchers, such as Allen and Feng [22], have derived non-dimensional parameters to characterize the structural response of the sandwich panel. These parameters can be used to guide an analyst in selecting a modeling fidelity for sandwich panel buckling analyses.

\section{Finite Element Modeling}

Sandwich panel finite element modeling has generally taken one of three approaches depending on panel geometry and constituent materials. The buckling behavior of a sandwich panel is dependent on the sandwich panel geometry and properties and may involve a general instability mode, a local instability mode, or an interaction between the two. Accurate prediction of general or overall instability modes requires adequate representation of the sandwich stiffnesses whereas prediction of local instabilities requires detailed through-the-thickness modeling. The first modeling approach exploits standard shell finite elements. These models are referred to herein as layered shell models, and for thin sandwich panels may provide a first approximation of the global behavior. The second approach uses standard shell finite elements for the face sheets and solid three-dimensional finite elements for the core. These models are referred to as layered shell/solid models and provide a modeling approach for both general and local response predictions. The accuracy of this approach, as well as its computational cost, is related to the through-the-thickness modeling of the core material. The third approach is a full threedimensional finite element model, in which solid threedimensional elements are used to model both the face sheets and the core. These models are referred to as three-dimensional solid models and they are typically reserved for detailed local modeling because of their computational cost. Recently, an additional modeling approach has been implemented in the STAGS nonlinear finite element code. This modeling approach, referred to herein as sandwich element models [18, 23], uses a specialty element developed specifically for the analysis of sandwich structures. Sandwich element models embody the kinematics and stiffness of the sandwich structure for less computational cost than the layered shell/solid models. Sandwich element models provide a cost-effective analysis approach for capturing sandwich behavior for large-scale sandwich structure simulations as well as for detailed local analyses. Details for these modeling approaches are described in the next sections.

\section{Layered Shell Models}

Layered shell models exploit the existing plate and shell finite element analysis features available in most finite element codes. The sandwich panel is modeled using two-dimensional shell elements with at least three groups of layers. The first group of layers corresponds to the laminate of one face sheet. The next group of layers corresponds to the core material, and the last group of layers represents the laminate of the other face 
sheet. This approach gives an equivalent single-layer result using classical lamination theory to compute the sandwich stiffness coefficients. In this model all layers have a common, unique rotation through the cross section of the sandwich. Because the core material generally only offers shear stiffness, shear-flexible $\mathrm{C}^{0}$ shell elements are typically used in layered shell models. Shell elements based on the classical Kirchhoff-Love theory ( $\mathrm{C}^{1}$ shell elements) can be used; however, such an approach ignores the transverse shear flexibility offered by the sandwich structure. Within STAGS, the 4-node $C^{1}$ shell element is called the 410 element (see [24]) and the 9-node ANS $\mathrm{C}^{0}$ shell element is called the 480 element (see $[25,26]$ ). Full integration is used for both shell elements (i.e., $2 \times 2$ and $3 \times 3$, respectively). Finite element formulations for sandwich panels based on high-order theories, such as Frostig [7] and Tessler et al. [8], are not available within STAGS and hence not included in this study.

\section{Layered Shell/Solid Models}

The next level of modeling uses shell elements to model each individual face sheet and solid elements to model the core material. These models are referred to herein as layered shel1/solid models. Each face sheet may be a multi-layer laminate and is modeled using shell elements, while the core is modeled using solid elements. Multiple solid elements through the core thickness may be required to represent the core deformation accurately. Displacement-field compatibility between the shell element and the solid element must be considered during the modeling process. For example, the use of 4-node $\mathrm{C}^{\mathrm{l}}$ shell elements for the face sheets with an 8-node solid element leads to an incompatibility for the normal displacements. Lagrangian shell elements based on a $\mathrm{C}^{0}$ formulation, used with standard solid elements of the same order, give displacement-field compatibility for the translational degrees of freedom. Combinations of the 4-node, 8-node or 9-node $\mathrm{C}^{0}$ shell element with the 8 node, 20-node or 27-node solid element, respectively, give translational displacement compatibility. Within STAGS, the compatible set of shell and solid elements are the 9-node ANS $\mathrm{C}^{0}$ shell element (480 element) and the 27-node ANS C $\mathrm{C}^{0}$ solid element ( 883 element). Full integration is used for both the shell and solid elements (i.e., $3 \times 3$ and $3 \times 3 \times 3$, respectively). For the layered shell/solid models, the shell element reference surface is defined to coincide with the bounding surface of the adjacent solid element. In addition, the rotational degrees of freedom of grid points associated with the core and not connected to the shell elements of the face sheets are constrained to zero.

\section{Sandwich Element Models}

A specialty finite element for sandwich panel analysis has been formulated by Riks and Rankin [23] and is described in the STAGS manual [18]. This specialty element exploits the existing shell finite element technology available in the finite element code itself. The deformation of the face sheets is modeled by using individual shell elements for each face sheet. Coupling between the two shell elements is carried out by applying an appropriate penalty function, consistent with the material behavior of the core, to enforce the kinematics of the core as a function of the kinematics of the face sheets. The core is assumed to have generally anisotropic three-dimensional elastic properties whose deformation is defined by the change in distance between adjacent face sheets. For this special sandwich element, the face sheets are intrinsically modeled using the standard STAGS 410 quadrilateral shell elements. Multiple sandwich elements may be stacked through the thickness of the core to provide a refined through-thethickness discretization of the core material wherein the intermediate face sheets are treated as "phantom" face sheets with zero thickness. Between adjacent face sheets, the transverse shear strain varies linearly with the thickness coordinate and the transverse normal strain is constant. The number of integration points through the core thickness of each sandwich element can be chosen to be either one or two depending on whether a spurious mode is triggered by the boundary conditions in the face sheets. Stiffer results are generally obtained when two integration points are used. Within STAGS, this specialty sandwich element is called the 840 element $[18,23]$. This modeling approach provides a computationally attractive approach for sandwich panel analysis for large-scale structures and also provides a capability to assess detailed response characteristics using a local strip model.

\section{Numerical Results and Discussion}

The basic geometry of the sandwich panels analyzed in this study is defined in Figure 2. The upper and lower face sheets are identical. Analytical predictions are made following the approach of Vinson [20] as implemented in PANDA2 [21]. Finite element analyses, using the STAGS nonlinear finite element analysis code [18], are conducted using layered shell models, layered shell/solid models, and sandwich element models. The layered shell models use the 4-node $C^{1} 410$ shell element and the 9-node ANS $\mathrm{C}^{0} 480$ shell element. The finite element approximation for the out-of-plane displacement is cubic for the 4-node element and quadratic for the 9-node element. For the layered shell/solid models, the face sheets are modeled using the 9-node ANS 480 shell element and the core is modeled 
using the 27-node ANS 883 solid brick elements through its thickness. This approach provides a compatible displacement field between the finite elements in the face sheets and those in the core. For the sandwich element models, the 840 sandwich elements $[18,23]$ are used through the core thickness.

Full panel models are defined as finite element models of the entire sandwich panel. Spatial discretization of the full panel is related to the anticipated buckling mode. Thus, some knowledge of the panel buckling response is needed to develop these full panel analysis models. For a rectangular panel, the number of half-waves, $m$, along the panel length in a general-instability panel-buckling mode is a function of the panel aspect ratio $a / b$, assuming a single half-wave across the width of the panel. Generally, five to six grid points per half-wave are required for accurate buckling predictions. To capture a face-sheet-wrinkling mode, the finite element mesh needs to be sufficiently refined to represent shortwavelength buckles.

Local strip models are defined as finite element models of a localized region with significant through-thethickness modeling detail so that short wavelength wrinkling modes can readily be detected. A local strip model is used to verify short-wavelength face-sheetwrinkling behavior predictions obtained with the full panel models. Local strip models represent a thin longitudinal slice of a panel away from edge effects. In the present study this strip model has a width equal to $2.54 \mathrm{~mm}$ and has 25 layers of sandwich elements through the core thickness. One integration point is used through the thickness of each core layer. The length of the local strip model depends on the wavelength of the buckling mode. Therefore, several local models, with different lengths, are analyzed to ensure that the lowest buckling load is determined.

Numerical results for two cases are presented. The first case considers a square sandwich panel with single-layer orthotropic face sheets and an isotropic core. This case studies the influence of different modeling approaches and boundary condition applications on the buckling response predictions for a given sandwich configuration loaded in uniaxial compression. The second case considers a rectangular sandwich panel with isotropic face sheets and an isotropic core. This case examines the influence of different modeling approaches on the buckling response predictions for sandwich panels with different core thickness and face-sheet thickness.

\section{Case 1: Square Sandwich Panel}

Several researchers $[13-15,17]$ have analyzed the buckling, under uniaxial compressive stress, of a simply supported $225-\mathrm{mm}$ square sandwich panel $(a / b=1)$ with identical single-layer orthotropic face sheets of a given fiber orientation angle and isotropic core. The face sheets have thickness $t_{f}$ equal to $0.2 \mathrm{~mm}$ and the core has thickness $h_{c}$ equal to $10 \mathrm{~mm}\left(h_{d} / a=0.044\right)$. The mechanical properties of the face-sheet material are: $E_{1}=229.0 \mathrm{GPa}, E_{2}=13.35 \mathrm{GPa}, G_{12}=5.25 \mathrm{GPa}$ and $v_{12}=0.315$ (i.e., $E_{2} / E_{1}=0.058$ ). Core material data are given in Table 1 . The fiber orientation of the face-sheet material is varied from zero to ninety degrees where the zero-degree orientation is parallel to the loading direction. For fiber orientation angles other than $0-$ and 90-degrees, the face sheets exhibit anisotropic behavior and the $D_{16}$ and $D_{26}$ bending stiffness terms become nonzero and large relative to the other bending stiffness terms. Consequently when the $D_{16}$ and $D_{26}$ bending stiffness terms become nonzero, the buckling mode shapes may become skewed, relative to the loading axis with non-straight modal node lines. In addition, as the face-sheet fiber-angle orientation increases, the number of half-waves in the general-instability mode increases. These complexities in the buckling mode shape impose additional modeling requirements on the analysis models over those required for the analysis of a panel without anisotropic effects.

The sandwich panel is loaded by a uniform end shortening, $u_{o}$, applied to the entire loaded edge (Edge 1 or $x=0$ in Figure 2). The in-plane pre-buckling stress state has uniform stress in the longitudinal direction, and the other two in-plane stress components are equal to zero. To achieve this pre-buckling stress state, the boundary conditions applied in the finite element models to all grid points along each edge are: $u=u_{0}$ and $w=\theta_{x}=\theta_{y}=\theta_{z}=0$ along Edge $1(x=0)$; $w=\theta_{x}=\theta_{y}=\theta_{z}=0$ along Edges 2 and $4(y=b$ and $y=0$, respectively); and $u=w=\theta_{x}=\theta_{y}=\theta_{z}=0$ along Edge $3(x=a)$. In addition, a point at the center of the panel has the transverse displacement $v$ set equal to zero to remove rigid-body motion. These boundary conditions result in a uniform stress state in the face sheets.

Having established a uniform uniaxial stress state, boundary conditions for the buckling analyses are defined next. All nodes through the thickness along the four edges of the sandwich panel are simply supported for the buckling calculations. These buckling boundary conditions are: $v=w=\theta_{x}=0$ along Edges 1 and 3 ( $x=0$ and $x=a$, respectively); and, $u=w=\theta_{y}=0$ along Edges 2 and 4 ( $y=b$ and $y=0$, respectively). For the layered shell modeling approach used in the present study, these simply supported boundary conditions are straightforward to impose on the finite element model. However, for the layered shell/solid modeling approach 
and the sandwich element modeling approach, independent approximations may be made for the bending rotations of each face sheet and core. In these approaches, the simply supported boundary conditions are defined so that shear deformations are prevented in the cross-sectional planes along the panel edges [15]. For the sandwich element modeling approach, the simply supported boundary conditions for buckling may be imposed using rigid links (multi-point constraints), along the boundaries, that tie the lower face sheet to the upper face sheet - similar to the kinematics relations of classical plate theory - when the pre-stress loading is an applied in-plane force and when constraints are employed to impose uniform end shortening. For the buckling calculations, the degrees of freedom associated with the end-shortening response are permitted to be free. For models with multiple sandwich elements through the core thickness, boundary conditions imposed along the panel edges at intermediate or "phantom" face sheets are the same as those imposed on the bounding face sheets.

Results of a convergence study conducted using the sandwich element modeling approach to predict the buckling response of a square sandwich panel with a 30 degree face-sheet fiber orientation angle are shown in Figure 3. The open symbols on dashed lines denote results obtained using models with one integration point (IP) through the thickness of the sandwich element, and the filled symbols on solid lines denote results obtained using models with two integration points. One, two, four, and eight layers of sandwich elements were analyzed for meshes with different levels of in-plane discretization. The predicted buckling loads are normalized by the solution obtained using the layered shell/solid approach with four solid elements through the core thickness and a $25 \times 25$ planar mesh of nodes (i.e., the buckling load used in the normalization is $340.0 \mathrm{~N} / \mathrm{mm}$ ). This solution obtained using the highest fidelity model is referred to herein as the reference solution and is denoted in Figure 3 by the " $x$ " symbol on a solid line. Hadi and Matthews [15] reported a value of $467.8 \mathrm{~N} / \mathrm{mm}$, and Yuan and Dawe [17] reported a value of $382.6 \mathrm{~N} / \mathrm{mm}$. Hadi and Matthews [15] used a Rayleigh-Ritz solution with trigonometric approximations for the in-plane variation and a zigzag theory through the thickness. Their results give a buckling solution stiffer than the present solution due to implicit boundary conditions from the displacement field approximations. Yuan and Dawe [17] used a finite strip solution with B-spline approximations for the inplane variation and the through-the-thickness approximations for the in-plane displacements are quadratic and the out-of-plane displacements are linear. Their results are much improved due to their treatment of the boundary conditions, yet still stiffer than the present reference solution that uses a piecewise quadratic approximation through the core thickness.

The predicted buckling mode shape is a generalinstability mode with a skewed single half-wave in both the $x$ and $y$ directions. As the number of grid points along each edge of the panel increases, the normalized buckling load converges from above, for all levels of the through-the-thickness discretizations and planar discretizations. In addition as the number of sandwich element layers increases, the convergence trends are well behaved, consistent, and approach the reference solution. The buckling load predicted using eight layers of sandwich elements is $5 \%$ higher than the reference solution obtained using the layered shell/solid approach. As the number of sandwich layers used to model the core thickness increases, the results are less sensitive to the number of integration points used through the thickness of each sandwich element. Use of one integration point gives a more flexible solution than the solution obtained using two integration points. However, when using one integration point, it is possible in some situations to trigger a spurious mode. In such cases, two integration points should be used.

Results of the present analysis approach, of Hadi and Matthews [15], and of Yuan and Dawe [17], expressed in terms of the in-plane stress resultant, are shown in Figure 4 as a function of the fiber orientation in the face sheet. The present results were generated using a full panel model with 25 grid points in each planar direction. These 625 grid points were used to define a finite element mesh of either 576 4-node quadrilateral shell elements or 169 9-node quadrilateral shell elements. Results of the present analysis are presented for layered shell models with and without shear deformation, layered shell/solid models, and sandwich element models. The results shown in Figure 4 indicate that the layered shell finite element models are stiffer and predict higher buckling loads than the layered shell/solid finite element model (reference solution). The results shown in Figure 4 indicate that the layered shell model without shear deformation (410 element models) provides a much stiffer solution (higher buckling loads) than the layered shell model with shear deformation (480 element models) and the buckling load decreases as the fiber angle increases from zero (results indicated by filled symbols). Results for the layered shell model with shear deformation (480 element models) indicate a more flexible solution (open symbol results) than that obtained using the 410 element. Results obtained for the sandwich element model (840 element models) with rigid links along the boundary edges, denoted by the " $x$ " symbol, indicate a response similar to that predicted using a layered shell model with shear deformation. The rigid links impose classical plate theory kinematics 
along the boundaries while internally the shear flexibility of the core material is included in the simulation. The buckling load is nearly constant up to a fiber angle of 30 degrees and then decreases as the fiber angles increases further.

The results shown in Figure 4 indicate that all finite element models of the present study and the analysis approach of Yuan and Dawe [17] predict a maximum buckling load when the face-sheet fiber orientation angle equals zero degrees and a progressive decrease in buckling load occurs as the fiber orientation angle increases. Hadi and Matthews [15] and additional earlier analyses $[13,14]$ using a Rayleigh-Ritz approach with trigonometric approximations, however, predict that the buckling load increases with increase in the face-sheet fiber angle from 0-degrees up to 40-degrees. Then the buckling load decreases rapidly to the buckling load value for the 90-degree case which is approximately half the buckling load value for the 0 degree case. This trend is similar to the trend obtained for a laminate composite plate with an aspect ratio greater than one (e.g., Ashton and Whitney [27]). However, for a square plate with these material properties, the buckling load decreases as the fiber angle increases. Yuan and Dawe [17] report a decrease in buckling load with an increase in fiber angle for the square sandwich panel under consideration and indicate that Rayleigh-Ritz solutions based on trigonometric series overly constrain the panel as material anisotropy increases. Double sine-series solutions for finite panels with simply supported boundary conditions and having symmetric laminates give rise to artificial derivative boundary constraints that prevent convergence to the correct solution [28, 29]. Stone and Chandler [29] report that buckling loads will be overestimated and may contribute to misleading trends or conclusions.

All finite element results from the present study indicate the same buckling mode shape (general instability) for any face-sheet fiber orientation. Initially $(0$-degree case) the mode shape involves only one half-wave in both directions. As the angle increases, the buckling mode shape becomes skewed and tends to follow the fiber angle. At approximately 60 degrees, the skewed mode shape has two longitudinal half-waves and one transverse half-wave. This pattern becomes less skewed as the face-sheet fiber angle approaches 90 degrees. In Figure 5, contour plots of the out-of-plane displacement component of the buckling mode shape are shown to illustrate this effect for different face-sheet fiber angles.

The effect of fiber angle on the buckling results obtained using the sandwich element modeling approach is shown in Figure 6. Results are shown for all models using one and four sandwich elements through the core thickness with each sandwich element having one or two integration points in the thickness direction (open and filled symbols, respectively). The finite element models of the full panel have 25 grid points in each planar direction. The present results bracket the solutions reported by Yuan and Dawe [17] and appear to be converging to the reference solution. As the number of sandwich elements through the core thickness increases, the sensitivity to the number of integration points decreases. These results illustrate the effectiveness of the STAGS sandwich element formulation.

Due to the nature of this problem and the differing results obtained by various researchers, two additional studies were performed to study the effect of the face sheet moduli ratio and panel aspect ratio on the general instability response of a sandwich panel. Results were obtained using the sandwich element modeling approach with four elements through the core thickness, and two integration points through the thickness of each element. Results for the present square panel with $E_{2} / E_{1}=0.058$ are shown in Figure 7 along with results for a rectangular panel with an aspect ratio, $a / b$, equal to two, and face-sheet moduli ratio $E_{2} / E_{1}=0.058$, and for a square panel with the transverse modulus, $E_{2}$, doubled $\left(E_{2} / E_{1}=0.116\right)$. Results for the square panels with $E_{2} / E 1=0.058$ and 0.116 are shown by the solid and dashed lines, respectively, and results for the rectangular panel are shown by the dotted line. As shown in Figure 7 , doubling the ratio of the face-sheet moduli ratio in the square panel causes an overall increase in the panel buckling load. Increasing the panel aspect ratio to two, while holding the face-sheet moduli ratio constant, results in a variation in the buckling load with face-sheet fiber angle similar to the variation obtained for a composite plate with similar material properties and an aspect ratio greater than one. For this case, the buckling load increases with an increase in face-sheet fiber angle up to 25 degrees, and then declines with increase in face-sheet fiber angle.

\section{Case 2: Rectangular Sandwich Panel}

The panel considered has a rectangular planform and is $508-\mathrm{mm}$ long and 254-mm wide $(a / b=2)$. Identical aluminum face sheets of thickness $t_{f}$ on an aluminum honeycomb core of thickness $h_{c}$ define the sandwich panel. Two face-sheet thicknesses are considered in this study: a thin face sheet with thickness equal to 0.508 $\mathrm{mm}$ and a thick face sheet with thickness equal to 2.794 $\mathrm{mm}$. The core thickness is varied from very thin, equal to the face-sheet thickness, to very thick, approaching half the panel width. As the core thickness increases, the buckling response transitions from a general instability mode to a short wavelength face-sheetwrinkling mode. Young's modulus, $E$, for the aluminum alloy is equal to $68.95 \mathrm{GPa}$ and Poisson's 
ratio is equal to 0.3 . The honeycomb core material is treated as homogeneous isotropic material with elastic mechanical properties given in Table 1. Comparative studies are performed for a specific panel geometry and material data. Analytical results are computed as the first level of analysis following Vinson's [20] approach as implemented in PANDA2 [21]. Finite element analyses are also conducted using STAGS. Finite element results are obtained using full panel models and local strip models. All modeling approaches are used in the full panel models, and only the sandwich element modeling approach is used in local strip models.

In the pre-stress condition, the sandwich panel is loaded by a uniform end shortening, $u_{o}$, applied to the sandwich face sheets on the ends of the panel at $x=0$ and $x=a$ (see Figure 2). For the buckling calculations, the boundary conditions applied to all grid points along each edge are: $u=u_{0}$ and $w=\theta_{x}=\theta_{z}=0 \quad$ along Edge $1 \quad(x=0)$; $w=\theta_{y}=0$ along Edges 2 and $4(y=b$ and $y=0$, respectively); and $u=-u_{0}$ and $w=\theta_{x}=\theta_{z}=0$ along Edge $3(x=a)$. In addition, a point at the center of the panel has $v=0$ to remove rigid body motion.

The finite element mesh for the full panel used in the present study has 81 grid points along the panel length and 21 grid points across the panel width. This spatial discretization (1,701 grid points) is held constant during the parametric studies and is adequate to represent buckling modes with up to sixteen half-waves along the panel length and four half-waves across the panel width. The mesh of 4-node elements (3,200 elements) has four times the number of elements as the mesh of 9 -node elements (800 elements). A layered shell modeling approach is attractive when general instability is anticipated because of the simplicity, ease of modeling and low computational cost of this modeling approach as compared to the other modeling approaches.

In the present study, the local strip model with a width equal to $2.54 \mathrm{~mm}$ and 25 sandwich elements through the core thickness is used to investigate face-sheet wrinkling. One integration point is used through the thickness of each sandwich element. The local strip model has a uniform stress state in the face sheets and buckling boundary conditions associated with a symmetric response. For small values of $h_{c} / a$, the PANDA2 wrinkling loads correlate well with those from the STAGS local strip model. For larger values of $h_{c} / a$, however, the PANDA2 wrinkling loads are much lower than those predicted by the STAGS model. Apparently the through-the-thickness assumptions made in PANDA2 are quite conservative. Based on the PANDA2 predictions, the core thickness that corresponds to a transition from a general-instability mode to a face-sheet-wrinkling mode is approximately $h_{d} / a=0.020$ for the thin face-sheet case and $h_{d} / a=0.060$ for the thick face-sheet case.

Linear buckling analysis results obtained from the full panel layered shell models indicate overall panel buckling regardless of the core thickness and the facesheet thickness. In all cases, the buckling mode corresponds to an overall panel mode with two halfwaves along the length and one half-wave across the panel width. In addition, the buckling results obtained using the 410 element are higher than the buckling results obtained using the 480 shear-flexible element, as expected. Good correlation between the layered shell model results and the analytical results from PANDA2 is obtained for sandwich structures with a very thin core. However, the finite element results based on the layered shell modeling approach quickly tend to deviate from the analytical results as the core thickness increases for a given panel length (increasing values of $h_{c} / a$ ) due to limitations in the layered shell models for modeling the core shear behavior and through-the-thickness flexibility.

Results obtained using the analytical model in PANDA2, the STAGS local strip wrinkling model, and the STAGS full panel layered shell/solid model are compared in Figure 8. PANDA2 wrinkling predictions are shown by the dotted line, the STAGS wrinkling model predictions are shown by the dashed line, and the STAGS full-panel layered shell/solid model predictions are shown by the symbols. In the layered shell/solid models, the core material is discretized using either one or four 27-node solid brick elements through the core thickness (open symbols and filled symbols, respectively, in Figure 8). PANDA2 overall panel buckling results are also shown as a solid curve in Figure 8. The results shown in Figure 8 indicate that the transition from an overall panel buckling mode to a wrinkling buckling mode occurs at a small value of the core thickness to panel length ratio $\left(h_{c} / a\right)$. In addition, these results show excellent agreement between the PANDA2 wrinkling results and the wrinkling results obtained with the local strip model for moderately thick sandwich panels (for ratio of core thickness to face-sheet thickness less than 50). For panels with a ratio of core thickness to face-sheet thickness greater than 50 , PANDA2 wrinkling results appear to be conservative, as a result of an assumed linear displacement response through the thickness of the core. The local strip model allows a piecewise linear distribution using 25 sandwich elements through the core thickness. In addition, the buckling results summarized in Figure 8 show that for the thin face-sheet case results obtained using the layered shell/solid models correlate well with the analytical results when just a single solid element 
through the core thickness is used. Good correlation is also shown between the results obtained using the local strip model and results obtained using the layered shell/solid model when four solid elements through the core thickness are used. For the thick face-sheet case with $t_{f}=2.974 \mathrm{~mm}$ and $h_{c} / a=0.4$, the layered shell $/ \mathrm{solid}$ models predict a buckling load that is approximately $8 \%$ higher than the buckling load predicted by the local strip model. The results for $t_{f}=2.974 \mathrm{~mm}$ and $h_{d} a=0.4$ indicate that the buckling load predicted by the layered shell/solid models is converging to $430.03 \mathrm{kN}$. The convergent behavior of the layered shell/solid modeling approach is evident even for short wavelength facesheet-wrinkling behavior with only a $2 \%$ change between the buckling load predictions obtained with one element and four elements through the core thickness.

Similar results for sandwich element models are summarized in Figure 9. The sandwich element models have the same discretization as the finite element models with 4-node quadrilaterals. In these models, the core material is discretized using either one or four sandwich elements through the core thickness with "phantom" face sheets for the intermediate layers (open symbols and filled symbols, respectively, in Figure 9). Results in Figure 9 show that for the thin face-sheet case, the sandwich element models with one integration point correlate well with the analytical results and with results obtained using the local strip model. Use of two integration points through the thickness of each sandwich element results in a stiffer response and higher buckling loads. Similar trends are observed for the thick face-sheet cases. However, as the core thickness increases, this convergent behavior is less evident. For example, results obtained using one integration point per layer for the thick face-sheet case and $h_{c} / a=0.4$, indicate an increase in buckling load as the number of layers increases from one to four. Further analyses conducted using six and eight sandwich elements through the thickness predict buckling loads of $432.99 \mathrm{kN}$ and $435.31 \mathrm{kN}$, respectively, thereby indicating a slow convergence. Use of two integration points per layer results in a very stiff solution for a single layer model, and the buckling load is nearly four times the value predicted with a four layer model. Additional analyses were performed using six and eight sandwich elements with predicted buckling loads of $479.28 \mathrm{kN}$ and 461.93 $\mathrm{kN}$, respectively. Again the results appear to be converging but extremely slowly for the case of two integration points per layer. These results indicate that the sandwich element modeling approach, while quite powerful for modeling large-scale structures exhibiting a general instability, is not effective in a large-scale sense for predicting face-sheet-wrinkling buckling modes unless local strip models are used.
Finally, a limited review of the computational cost associated with the sandwich element and layered shell/solid element modeling approaches was performed for the thin face-sheet case with $h_{c} / a=0.1$. The sandwich element approach exploits the 4-node $\mathrm{C}^{1}$ shell element formulation while the layered shell/solid element approach is based on the ANS formulation for a 9-node $C^{0}$ shell element and a 27-node solid element. For a fixed finite element discretization (same number of grid points) in the plane of the sandwich panel, the critical buckling load for the sandwich element modeling approach changes from $52.1 \mathrm{kN}$ to $67.6 \mathrm{kN}$ to $76.8 \mathrm{kN}$ as the number of through-the-thickness layers increases from one to two to four, respectively. Similarly, the layered shell/solid modeling approach gives critical buckling loads that change from $77.7 \mathrm{kN}$ to $75.2 \mathrm{kN}$ to $73.7 \mathrm{kN}$ as the number of through-thethickness layers increases from one to two to four, respectively. Clearly for this wrinkling mode response, the layered shell/solid modeling approach converges more quickly than the sandwich element modeling approach. The advantage of using the sandwich element modeling approach from a computational cost perspective decreases as the number of sandwich element layers increases. The computational effort associated with a sandwich element model is defined in terms of the number of through-the-thickness layers which in turn increases the size of the global stiffness matrix. The number of equations in the global stiffness matrix for a model with a single layer of sandwich elements is approximately $60 \%$ of that for a layered shell/solid model with a single solid element in the core. As the number of layers of sandwich elements increases, the number of equations increases proportionally in addition to the computational effort expended at the element level. Buckling values obtained using either approach with four layers through the thickness are nearly equal. However, the solutions obtained with a single layer of sandwich elements require only $60 \%$ of the computational effort required to obtain a solution with a single solid element in the core. Approximately the same level of solution accuracy is achieved by using a single solid element in the core of a layered shell/solid model as is obtained by using four layers of the sandwich elements. In this case, the computational cost for the sandwich element modeling approach is over four times the computational cost of the layered shell/solid model to achieve equal levels of solution accuracy. Extrapolation of this cost differential to largescale structural panels is not expected to apply because of solid element aspect ratio limits. However, use of the sandwich element modeling approach for large-scale sandwich structures provides an effective means to determine its general-instability buckling response. 


\section{Conclusions}

A comparative study of different modeling approaches for predicting sandwich panel buckling response has been presented. The study considered sandwich panels with anisotropic face sheets and a very thick core. Results from conventional analytical solutions for sandwich panel overall buckling and face-sheetwrinkling type modes were compared with solutions obtained using different finite element modeling approaches. The numerical analyses were conducted using the STAGS nonlinear finite element code, and the analytical results were computed using PANDA2. Finite element solutions were obtained using layered shell element models, with and without transverse shear flexibility, layered shell/solid element models, that use shell elements for the face sheets and solid elements for the core, and sandwich models using a recently developed specialty sandwich element. Convergence characteristics of the shell/solid and sandwich element modeling approaches with respect to in-plane and through-the-thickness discretization, were demonstrated.

Results of the study indicate that the specialty sandwich element formulation implemented in STAGS provides an accurate and effective modeling approach for predicting both overall and localized behavior. This modeling approach provides the flexibility for modeling an entire panel or for modeling local detail through the core thickness. Results of the study indicate that transition from a general instability mode to a short wavelength face-sheet-wrinkling mode occurs as the core thickness is increased. Modeling fidelity to capture both possible modes must be provided near the transition region. Furthermore, results indicate that anisotropy of the face sheets, along with the ratio of principle elastic moduli, significantly affect the buckling response, and these effects may not be accurately represented by analytical solutions.

\section{Acknowledgement}

The work of the second, third and fourth authors was supported by the NASA Langley Research Center under contracts NAS1-00135, GS-35F-0038J (Task 1730), and NAS1-99069, respectively.

\section{References}

1. Allen, Howard G., "Sandwich Construction Today and Tomorrow," in Sandwich Construction 1, KarlAxel Olsson and Ronnal P. Reichard (editors), Proceedings of the First International Conference on Sandwich Constructions, Stockholm, Sweden, June 19-21, 1989, pp. 3-22.

2. Plantema, F. J., Sandwich Construction, John Wiley, New York, 1966.

3. Anon., Structural Sandwich Composites, MilHDBK-23A, Department of Defense, Washington, D.C., 1968.

4. Benson, A. S. and Mayers, J. “General Instability and Face Wrinkling of Sandwich Plates - Unified Theory and Applications," AIAA Journal, Vol. 5, No. 4, April 1967, pp. 729-739.

5. Ha, K. H., "Finite Element Analysis of Sandwich Construction: A Critical Review," in Sandwich Construction 1, Karl-Axel Olsson and Ronnal P. Reichard (editors), Proceedings of the First International Conference on Sandwich Constructions, Stockholm, Sweden, June 19-21, 1989 , pp. 69-85.

6. Ha, K. H., "Finite Element Analysis of Sandwich Plates: An Overview," Computers and Structures, Vol. 37, No. 4, 1990, pp. 397-403.

7. Frostig, Y., "Buckling of Sandwich Panels with a Flexible Core - High-Order Theory," International Journal of Solid and Structures, Vol. 35, Nos. 3-4, 1998, pp. 183-204.

8. Tessler, A., Annett, M. S., and Gendron, G., "A $\{1,2\}$-Order Plate Theory Accounting for ThreeDimensional Thermoelastic Deformations in Thick Composites and Sandwich Laminates," Composite Structures, Vol. 52, 2001, pp. 67-84.

9. Vonach, W. K. and Rammerstorfer, F. G., "A General Approach to the Wrinkling Instability of Sandwich Plates," Structural Engineering and Mechanics, Vol. 12, No. 4, 2001, pp. 363-376.

10. Bert, C. W., "Shear Deformation and Sandwich Configuration," in Buckling and Postbuckling of Composite Plates, G. J. Turvey and I. H. Marshall (editors), Chapman and Hall, 1995, pp. 157-189.

11. Noor, A. K., Burton, W. S., and Bert, C. W., "Computational Models for Sandwich Panels and Shells," Applied Mechanics Reviews, Vol. 49, No. 3, March 1996, pp. 155-199.

12. Librescu, L. and Hause, T., "Recent Developments in the Modeling and Behavior of Advanced Sandwich Constructions: A Survey," Composite Structures, Vol. 48, Nos. 1-3, January-March 2000, pp. $1-17$. 
13. Rao, K. M., "Buckling Analysis of Anisotropic Sandwich Plates Faced with Fiber-Reinforced Plastics," AIAA Journal, Vol. 23, No. 8, August 1985, pp. 1247-1253.

14. Kim, C. G. and Hong, C. S., "Buckling of Unbalanced Anisotropic Sandwich Plates with Finite Bonding Stiffness," AIAA Journal, Vol. 26, No. 8, August 1988, pp. 982-988.

15. Hadi, B. K. and Matthews, F. L., "Predicting the Buckling Load of Anisotropic Sandwich Panels: An Approach Including Shear Deformation of the Faces," Composite Structures, Vol. 42, No. 3, July 1998, pp. 245-255.

16. Dawe, D. J. and Yuan, W. X., "Overall and Local Buckling of Sandwich Plates with Laminated Faceplates, Part I: Analysis," Computer Methods in Applied Mechanics and Engineering, Vol. 190, 2001, pp. 5197-5213.

17. Yuan, W. X. and Dawe, D. J., "Overall and Local Buckling of Sandwich Plates with Laminated Faceplates, Part II: Applications," Computer Methods in Applied Mechanics and Engineering, Vol. 190, 2001, pp. 5215-5231.

18. Rankin, C. C., Brogan, F. A., Loden, W., and Cabiness, H., Structural Analysis of General Shells - STAGS User Manual, Version 4.0, Lockheed Martin Advanced Technology Center, Palo Alto, CA, June 2000

19. Brush, D. O. and Almroth, B. O., Buckling of Bars, Plates and Shells, McGraw-Hill, New York, 1975.

20. Vinson, J. R., "Optimum Design of Composite Honeycomb Sandwich Panels Subjected to Uniaxial Compression," AIAA Journal, Vol. 24, No. 10, October 1986, pp. 1690-1696.

21. Bushnell, David "Optimum Design via PANDA2 of Composite Sandwich Panels with Honeycomb or Foam Cores," AIAA Paper No. 97-1142, April 1997.

22. Allen, H. G. and Feng, Z., "Classification of Structural Sandwich Panel Behavior," in Mechanics of Sandwich Structures, A. Vautrin (editor), Proceedings of the EUROMECH 360 Colloquium held in Saint-Etienne, France, 13-15 May 1997, Kluwer Academic Publishers, Dordrecht, 1998, pp. $1-12$.

23. Riks, E. and Rankin, C. C., "Sandwich Modeling with an Application to the Residual Strength Analysis of a Damaged Composite Compression Panel," AIAA Paper 2001-1323, April 2001.

24. Rankin, C. C. and Brogan, F. A., The Computational Structural Mechanics Testbed Structural Element Processor ES5: STAGS Shell Element, NASA CR-4358, 1991.
25. Stanley, G. M., The Computational Structural Mechanics Testbed Structural Element Processor ES1: Basic SRI and ANS Shell Elements, NASA CR-4357, 1990.

26. Park, K. C. and Stanley, G. M., "A Curved C Shell Element Based on Assumed Natural-Coordinate Strains," ASME Journal of Applied Mechanics, Vol. 108,1986 , pp. $278-290$.

27. Ashton, J. E. and Whitney, J. M., Theory of Laminated Plates, Technomic Publishing Co., 1970.

28. Dawe, D. J. and Peshkam, V., "Buckling and Vibration of Finite-Length Composite Prismatic Plate Structures with Diaphragm Ends, Part I: Finite Strip Formulation," Computer Methods in Applied Mechanics and Engineering, Vol. 77, Nos. 1-2, December 1989, pp. 1-30.

29. Stone, M. A. and Chandler, H. D., "Errors in Double Sine Series Solutions for Simply Supported Symmetrically Laminated Plates," International Journal of Mechanical Sciences, Vol. 38, No. 5, 1996, pp. 517-526.

Table 1. Elastic mechanical properties for core materials

\begin{tabular}{ccc}
\hline \hline Material Properties & Case 1 & Case 2 \\
\hline $\mathrm{E}_{\mathrm{cx}}=\mathrm{E}_{\mathrm{cy}}, \mathrm{MPa}$ & 200 & 0.6895 \\
$\mathrm{E}_{\mathrm{cz}}, \mathrm{MPa}$ & 200 & 68.95 \\
$\mathrm{G}_{\mathrm{cxy}}, \mathrm{MPa}$ & 146 & 0.265 \\
$\mathrm{G}_{\mathrm{cxz}}, \mathrm{MPa}$ & 146 & 82.74 \\
$\mathrm{G}_{\mathrm{cyz}}, \mathrm{MPa}$ & 90.4 & 49.64 \\
$\mathrm{v}_{\mathrm{cxy}}$ & 0.3 & 0.3 \\
$\mathrm{v}_{\mathrm{cxz}}=\mathrm{v}_{\mathrm{cyz}}$ & 0.3 & 0.01 \\
\hline \hline
\end{tabular}




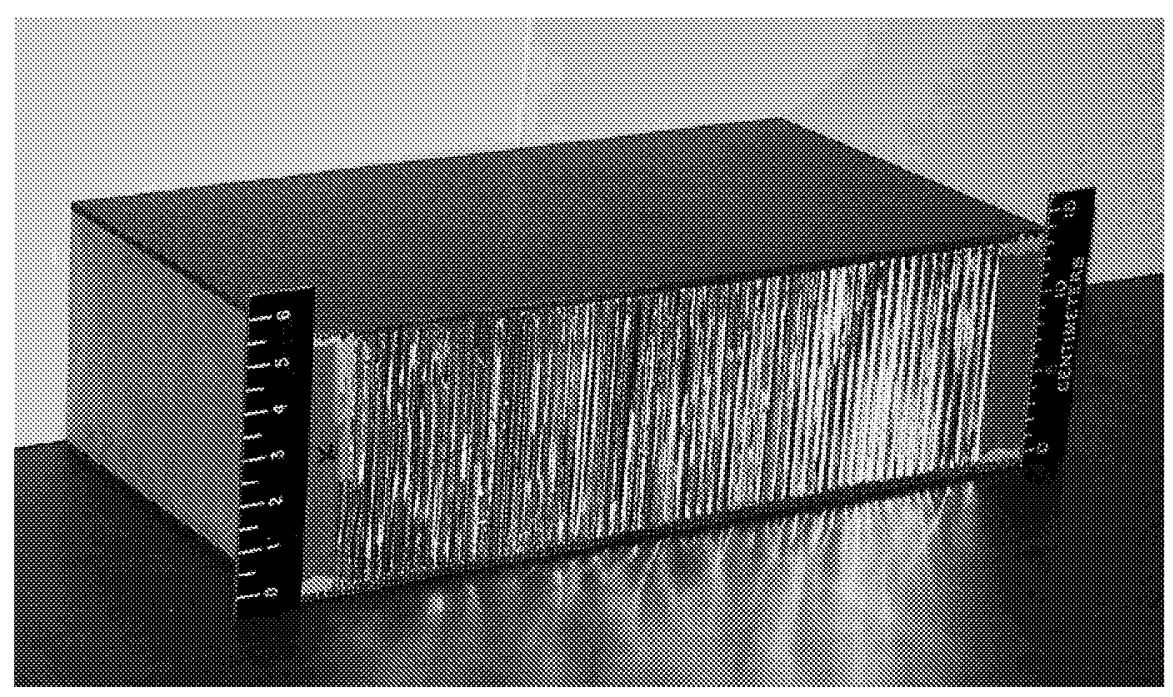

Figure 1. Photograph of thick sandwich panel segment.
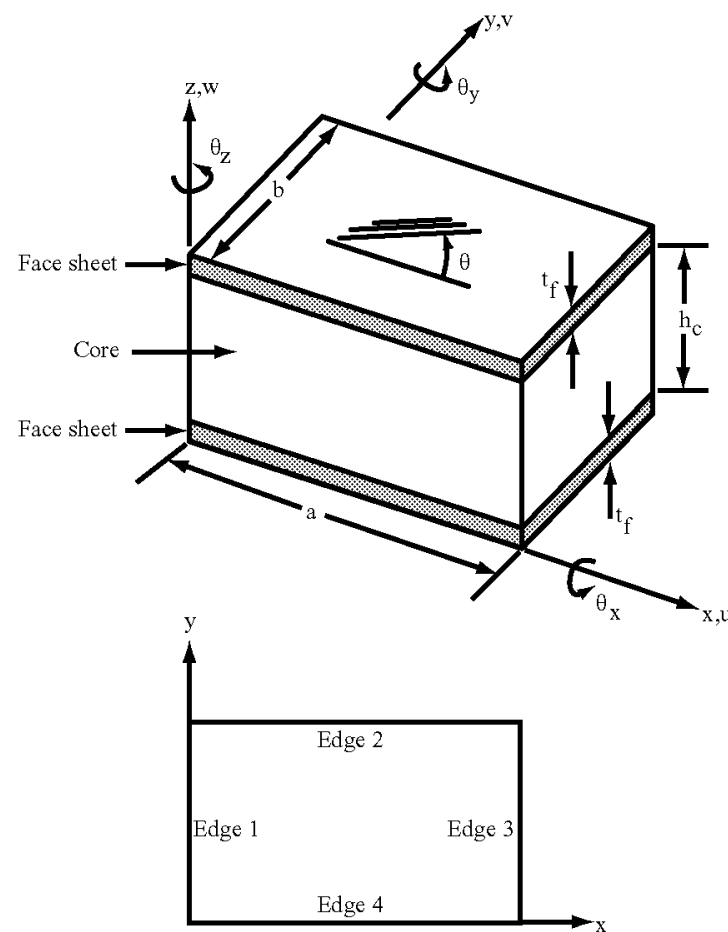

Figure 2. Sandwich panel geometry. 


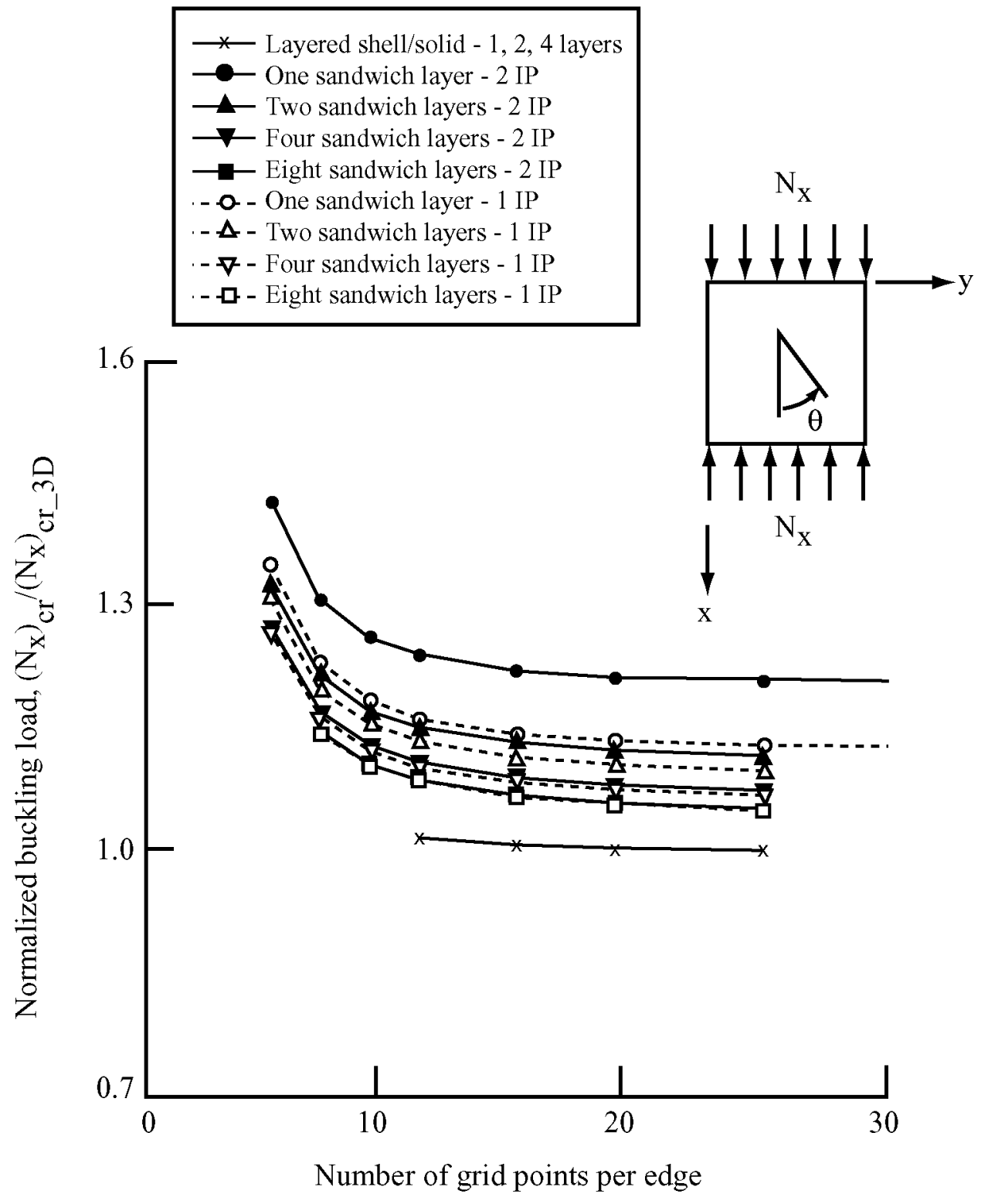

Figure 3. Effect of mesh refinement for sandwich element models for the square sandwich panel with a 30degree fiber angle. 


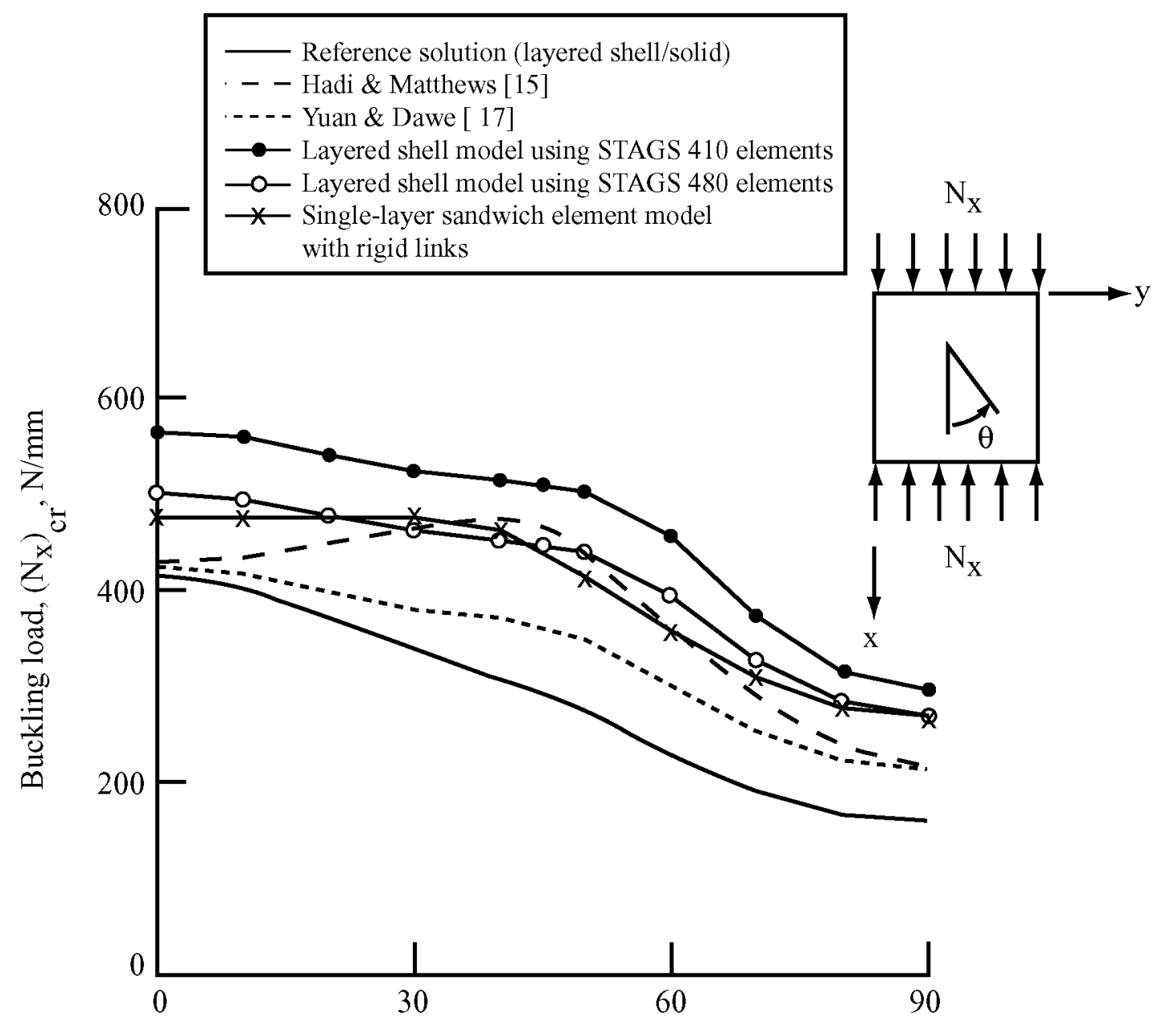

Ply layup angle, $\theta$, degrees

Figure 4. Effect of face-sheet fiber angle on buckling load for layered shel1/solid and layered shell models of the square sandwich panel. 


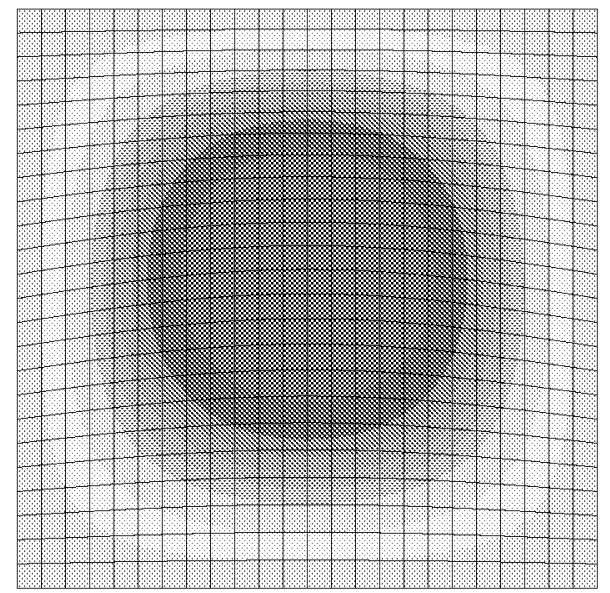

a) 0-degree case

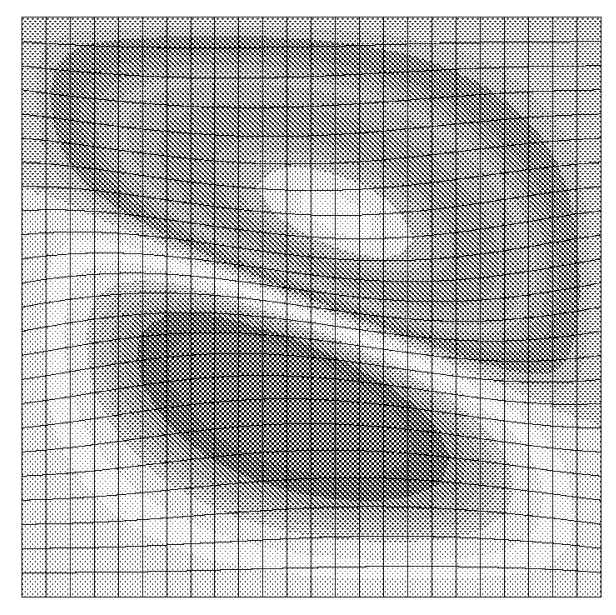

c) 60-degree case

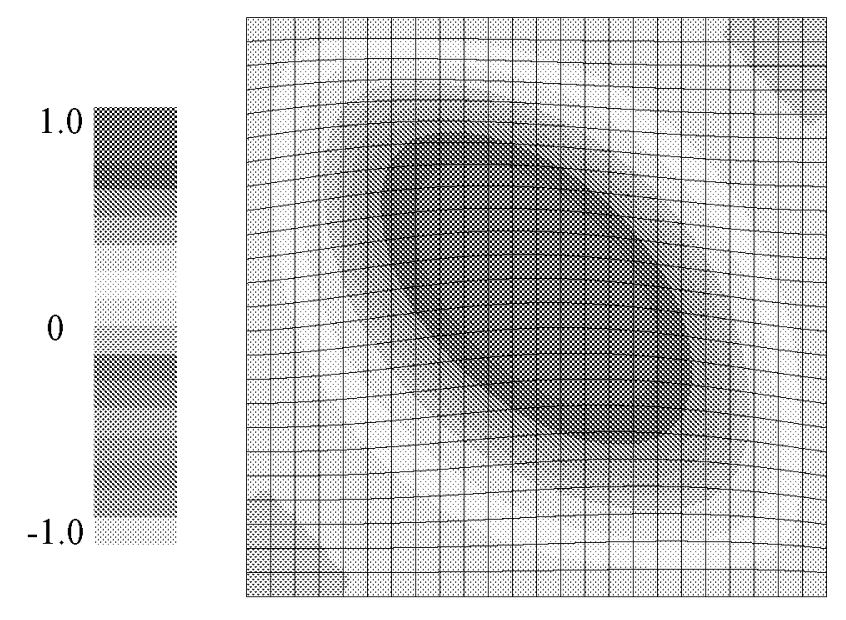

b) 40-degree case

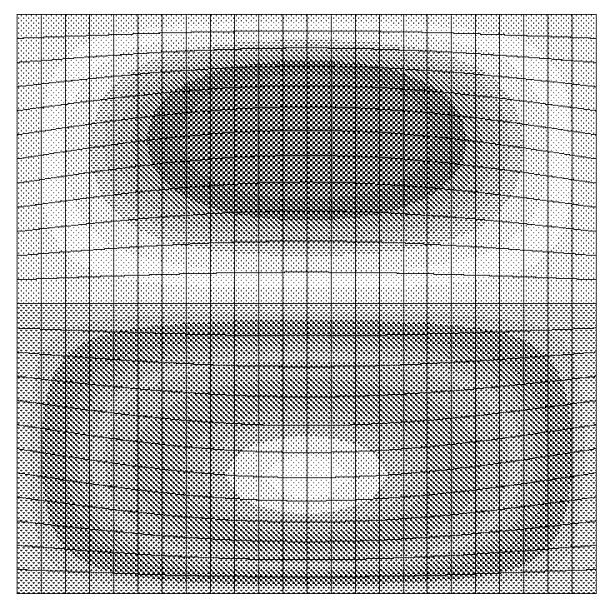

d) 90-degree case

Figure 5. Contour plots of the out-of-plane displacement component of the buckling mode shape for different face-sheet fiber angles. 


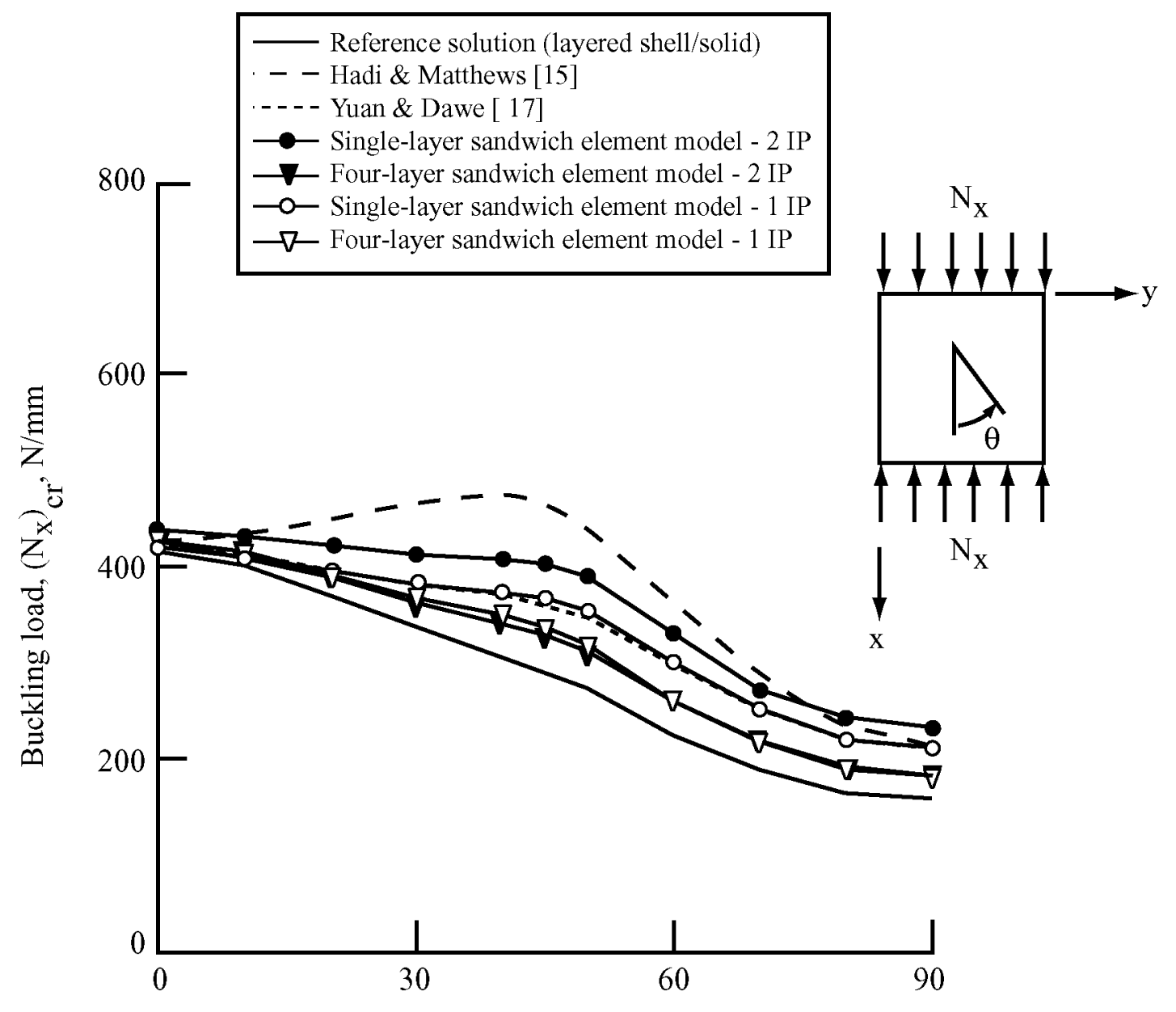

Ply layup angle, $\theta$, degrees

Figure 6. Effect of face-sheet fiber angle on buckling load for layered shel1/solid model and sandwich element models of the square sandwich panel. 


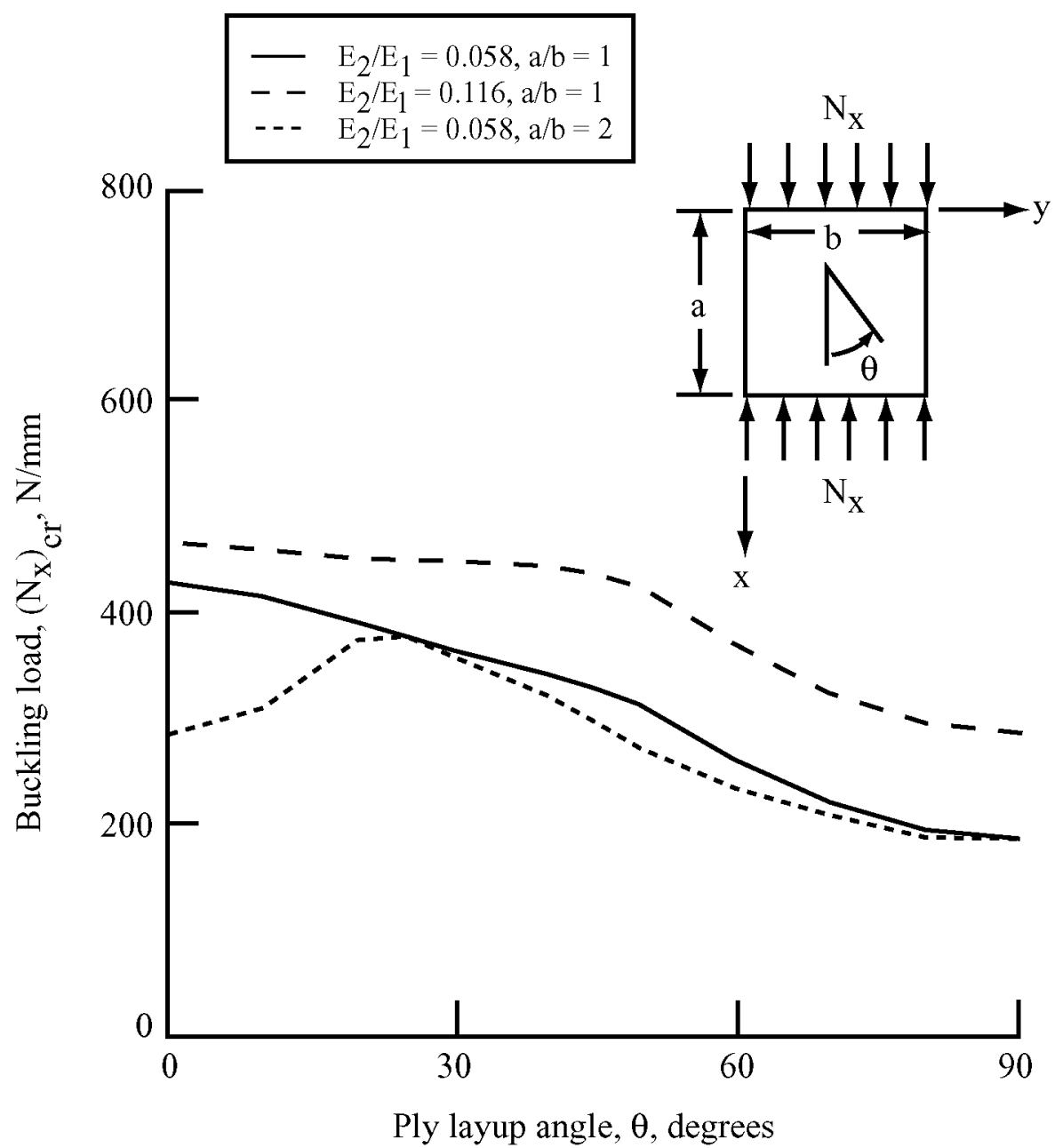

Figure 7. Effect of face-sheet fiber angle, aspect ratio, and face-sheet elastic modulus ratio $\left(\mathrm{E}_{2} / \mathrm{E}_{1}\right)$ on buckling load for a sandwich element model of the square sandwich panel. 


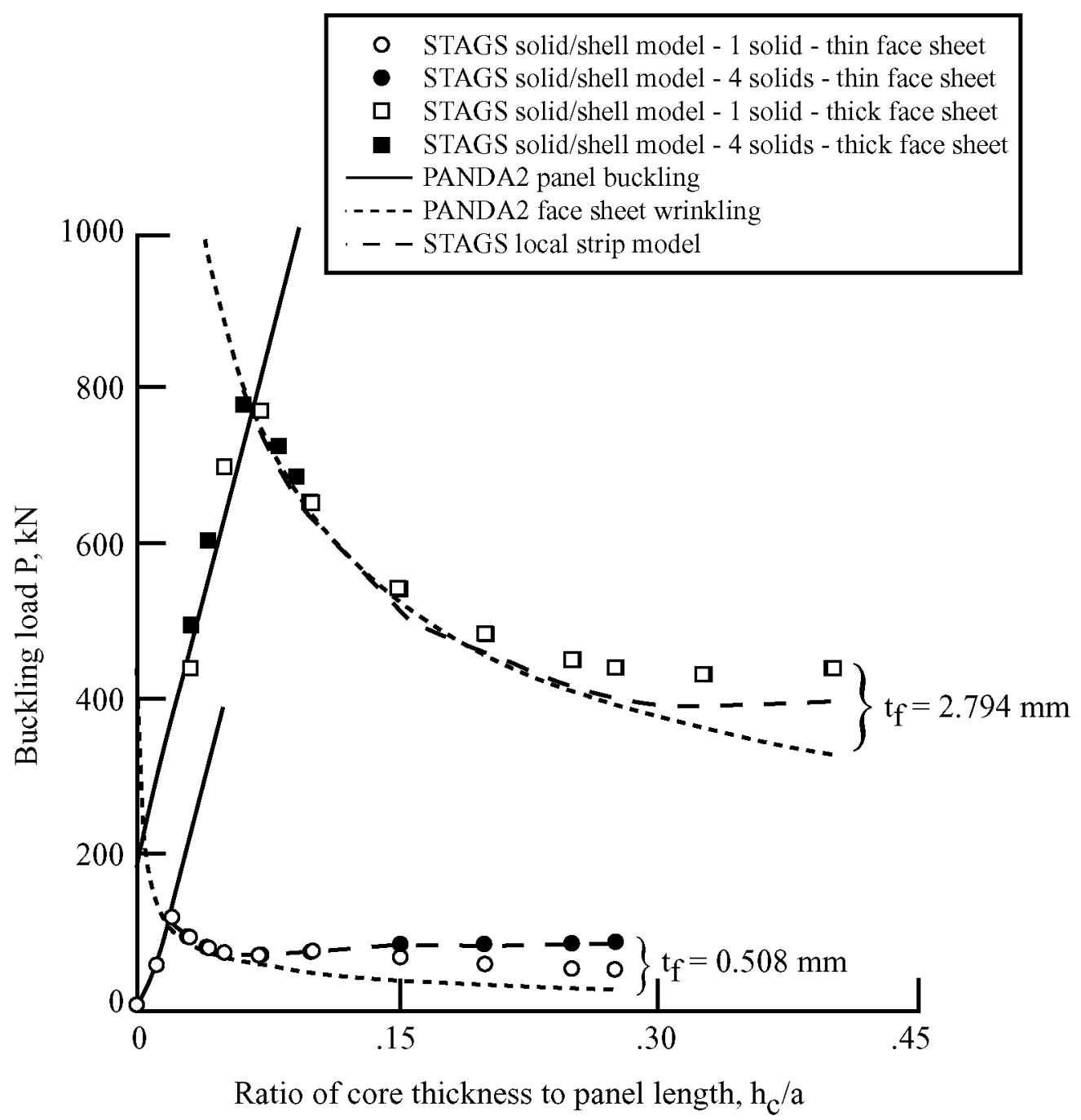

Figure 8. Buckling load predictions from analytical model, local strip sandwich element model and full panel layered shell/solid model. 


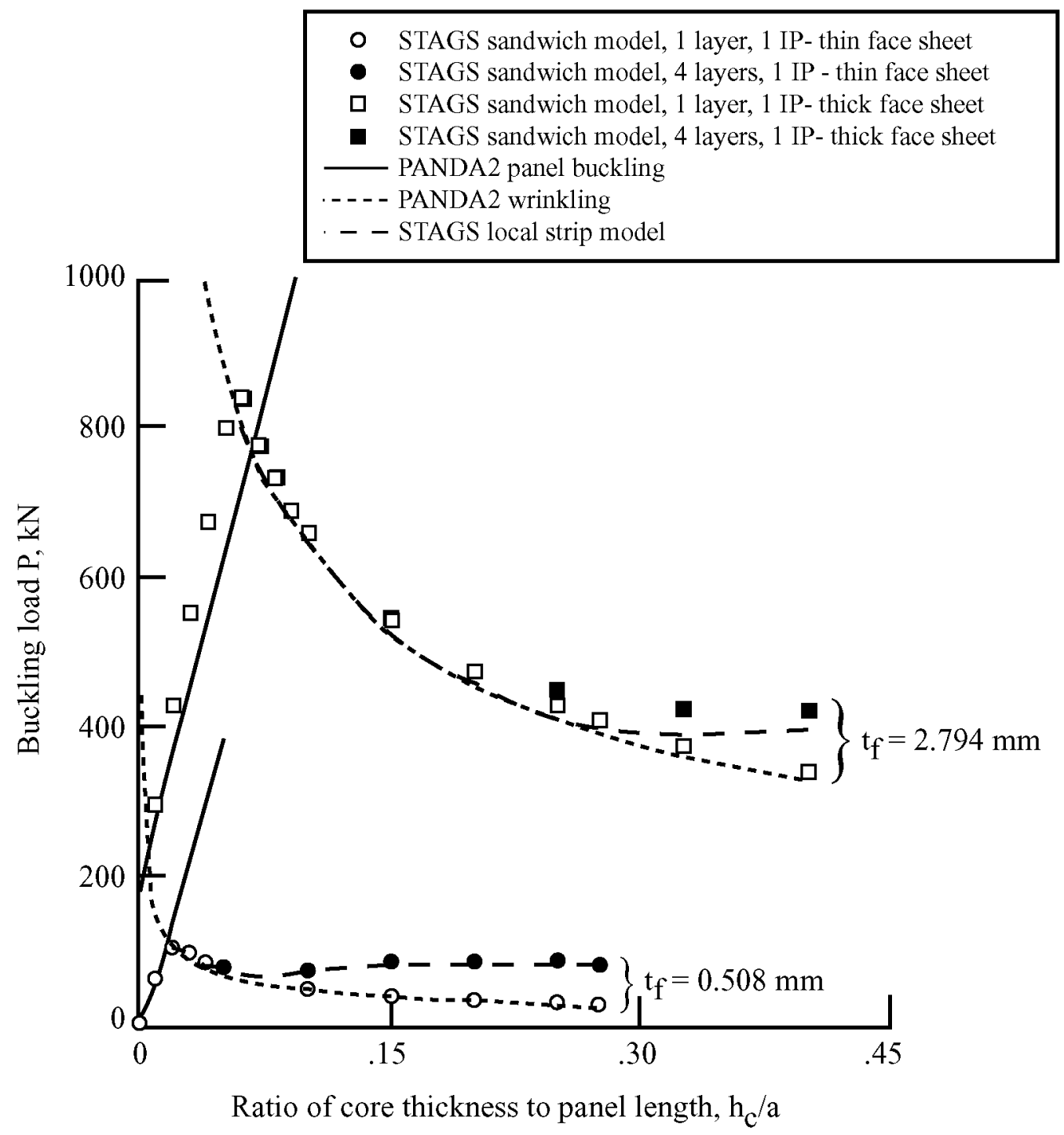

Figure 9. Buckling load predictions from analytical model, local strip sandwich element model and full panel sandwich element model. 